\title{
Investigation and Analysis of the Sub-health Status of the Members of the Medical Team Aiding Wuhan During the Novel Coronavirus Pneumonia (COVID- 19) Outbreak
}

\section{Meixian Zhang}

Guangzhou Special Service Recuperation Center of PLA Rocket Force Haoyuan Chen

Guangzhou Special Service Recuperation Center of PLA Rocket Force Chen Huang

School of Public Health,Southern Medical University

Xia Li ( $D 717163146 @ q q . c o m$ )

Guangzhou Special Service Recuperation Center of PLA Rocket force

Dihui Xu

Guangzhou Special Service Recuperation Center of PLA Rocket Force Jie Li

Guangzhou Special Service Recuperation Center of PLA Rocket Force

Qian Liu

School of Health Service Management, Southern Medical University Jun Xu

Department of Sanitation Economy Administration, Nanfang Hospital ,Southern Medical University

Research

Keywords: sub-health, novel coronavirus pneumonia, military medical team aiding Wuhan

Posted Date: September 17th, 2020

DOl: https://doi.org/10.21203/rs.3.rs-74743/v1

License: (c) (i) This work is licensed under a Creative Commons Attribution 4.0 International License. Read Full License 


\section{Abstract}

Objective: To understand the sub-health status of the medical team members of the aid to Wuhan during the outbreak of the new coronavirus pneumonia in Wuhan, China, to analyze the possible influencing factors that cause the sub-health status, and to provide a theoretical basis for scientifically maintaining and improving the physical and mental health of the medical team members.

Methods: 131 members of the military medical team aiding Wuhan who were treating patients with novel coronavirus pneumonia were selected as subjects in the survey, and a questionnaire on the sub-health influencing factors was designed. After the medical treatment task, the SHMS V1.0 was used for assessment.

Results: The sub-health detection rate of the medical team members was $76.3 \%$, and the overall subhealth rating scale score was $(69.11 \pm 10.20)$ points. The scores of the three subscales of physiology, psychology, and society are $(71.16 \pm 11.30)$ points, $(67.99 \pm 13.51)$ points, and $(67.41 \pm 12.31)$ points, respectively. Work pressure, family structure, frustration, and diet may be the influence factors of the subhealth.

Conclusion: During the outbreak of the novel coronavirus pneumonia, factors such as high-intensity work, non-nuclear families, low hobbies, and irregular diet may lead to the sub-healthy state of the members of the medical aid team in Wuhan. Reasonable arrangements for work and rest, maintaining harmonious family relations, focusing on the development of interests and hobbies, and healthy and regular diet, will help maintain and improve the health of medical team members, consolidate and maintain the combat effectiveness of medical team members, and complete epidemic prevention and control tasks with high quality and efficiency.

\section{Introduction}

In December 2019, a novel coronavirus pneumonia (Coronavirus Disease 2019, COVID-19) ${ }^{[1]}$ outbreak occurred in Wuhan City, Wuhan Province, China, and it has been spreading rapidly throughout the country. In order to scientifically contain the spread of the pandemic and increase the cure rate of pneumonia, medical teams from all over the country actively responded to the call and rushed to help Wuhan. Upon hearing the order, the People's Army dispatched a medical team to assist Wuhan to quickly stationed in Wuhan and became the main force in the fight against the pandemic. It took 84 days of heroic battle and finally completed the medical rescue mission successfully and achieved the predetermined goal of "winning the war with zero infection".

The novel coronavirus pneumonia is highly infectious, and the latest research reported that its basic reproduction number (RO) is as high as $5.7^{[2]}$. In the early stage of the pandemic, medical staff involved in the frontline treatment are facing many unknown risks in a high-risk environment of occupational exposure. They are shouldering the heavy responsibility of rescuing the dying and healing the wounded, and at the same time being under strong mental pressure, which is detrimental to their physical and 
mental health. These can easily lead to sub-health status, which will reduce work efficiency, weaken the rescue force, and bring adverse effects on the epidemic prevention and control. To this end, we conducted a preliminary analysis of the sub-health status of the medical team, and screened possible influencing factors, aiming to further improve the occupational protection of the medical team aiding Wuhan, improve the treatment capabilities, and then help maintain and improve the physical and mental health of the medical team on a scientific basis.

\section{Objects And Methods}

1.1 Subjects A total of 152 members of the military medical team who have performed a frontline rescue mission for Wuhan since January 24, 2020 were selected as subjects, and a questionnaire has been issued at the end of the mission.

\subsection{Methods}

1.2.1 Collection of basic information. During the COVID-19 outbreak in Wuhan, health conditions and professional characteristics of the members of the medical team aiding Wuhan were collected, including basic personal information, lifestyle and experience events. In detail, personal information includes gender, age, education level, marital status, job category, job title, personal monthly income, etc.; lifestyle and experience events are comprised of sleep status in the last month, work status, contact frequency with family members, work pressure, family structure, physical health, etc.

1.2.2 Application of Sub-Health Measurement Scale Version 1.0 (SHMS V1.0) ${ }^{[3]}$. The scale includes 3 sub-scales of physiological sub-health (SH1-SH14) scale, mental sub-health (SH16-SH27) scale, and social sub-health (SH29-SH37) scale, consisting of 9 dimensions (including Physical symptoms, organ functions, physical motor functions, energy, positive emotions, psychological symptoms, cognitive functions, social adaptation, social resources and social support). They are consisting of a total of 39 items (SH1-SH39), with good reliability and validity ${ }^{[3-4]}$. SHMS V1.0 scoring standard adopts the internationally accepted Likert five-level scoring method, and the scores of forward scoring items (including question 1-3, 13-19, 26-39) are the same as the original (1-5 points); The score of reverse scoring items (including question $4-12$ and 20-25) is equal to 6 minus the original score ${ }^{[2]}$. The sum of the scores of the items contained in each dimension is the original rough score of the dimension (labeled $\mathrm{P} 1, \mathrm{P} 2, \mathrm{P} 3, \mathrm{P} 4, \mathrm{M} 1, \mathrm{M} 2, \mathrm{M} 3, \mathrm{~S} 1, \mathrm{~S} 2)$; the sum of the scores of the dimensions contained in each subscale is the original rough score of the subscale (labeled PS, MS, SS, respectively); the sum of the scores of the three subscales is the original rough score of the total scale (labeled GS), the higher the score, the better the health ${ }^{[3]}$. This study uses conversion scores for the analysis of the sub-health scale and subscales. The formula is as follows (take a dimension as an example):Dimensional conversion score $=$ (original rough score of the dimension - lowest score of the dimension in theory) / (highest score of the dimension in theory - lowest score of the dimension in theory) $\times 100 \%$.

\subsubsection{Quality control}


This study collects data by issuing questionnaires. Before filling out the questionnaire, the purpose and method of filling in the form were explained to the tester, and then the tester were asked to select the options of true feeling anonymously based on his/her own situation in the last 4 weeks. In order to ensure the quality of the survey data, the returned questionnaires are screened, and those with a completion rate of less than $80 \%$, low-quality fill-in (such as the same answer for the entire questionnaire) and two completely duplicate questionnaires are excluded.

\subsubsection{Statistical methods}

EpiData3.02 software was used for double data entry and verification, and SPSS20.0 statistical software was used for data analysis. Measurement data is presented as ( $x \pm s)$, and count data as (\%). T-tests of two independent samples are used for comparison between two groups, and one-way analysis of variance is used for comparison between multiple groups (Brown-Forsythe method is used for the test when the variance is uneven); multiple linear regression analysis was used to analyze the influencing factors of the sub-health status of the medical team aiding Wuhan. The difference is statistically significant when $\mathrm{P}<0.05$.

\section{Results}

\subsection{Analysis of the basic situation of the medical team members}

A total of 152 sub-health questionnaires were issued and 132 were recovered, with a recovery rate of $86.84 \%$, of which 131 were valid questionnaires with an effective rate of $99.24 \%$. The basic information is shown in Table 1.

\subsection{The scores of the (SHMS V1.0 of the medical team aiding Wuhan}

The overall score of the sub-health assessment scale for the medical team in Wuhan is $(69.11 \pm 10.20)$ points. The three subscale scores are: physiological subscale score $(71.16 \pm 11.30)$ points, psychological subscale score $(67.99 \pm 13.51)$ points, social subscale score $(67.41 \pm 12.31)$ points. The scores of each dimension are shown in Table 2.

\subsection{Single factor analysis of the sub-health status of the medical team aiding Wuhan}

The total scores of the female mental sub-health subscale were significantly lower than the total scores of the male $(P<0.05)$. Age has no obvious influence on the scores of each scale $(P>0.05)$. Among the groups of different education levels, the scores of the sub-scale of physiological sub-health of the vocational school or high school group and the undergraduate group were significantly higher than the scores of the sub-scale of physiological sub-scale of the junior college, master's degree, and doctoral degree groups $(P<0.05)$. The overall sub-health status of the unmarried and divorced groups is better, and their mental sub-health and social sub-health subscale scores are higher than those of the married, widowed and unmarried cohabitation groups $(P<0.05)$. Departments, positions, working years, title and 
monthly income had no significant influence on the scores of each scale $(P>0.05)$. The scores of each subscale are shown in Table 3.

Work pressure, only child, family structure, anti-frustration quotient, hobbies, irregular diet, dietary bias, betrayal, and children's education problems have statistically significant differences in the total subhealth scores $(P<0.05)$. Sleep duration has a certain effect on physiological sub-health $(P<0.05)$. Daily working time affects mental sub-health significantly $(P<0.05)$. Except for betrayal, overtime, night shift, contact with family members, diagnosed diseases, and emergency response had no significant impact on the scores of each scale $(P>0.05)$. See Table 4.

\subsection{Multifactorial comparative analysis of the sub-health status of the medical team aiding Wuhan}

Taking the total score of the SHMS V1.0 of the medical team aiding Wuhan as the dependent variable, and taking 9 factors including work pressure, only child, family structure, anti-frustration quotient, hobbies, irregular diet, dietary bias, betrayal, and children's education problems as the independent variables, multiple linear regression analysis was performed (entry standard $a=0.05$, exclusion standard $\beta=0.10$ ). As a result, it is shown that 6 factors including work pressure, frustration, hobbies, dietary bias, betrayal, children's education problems, etc. are the main influencing factors of sub-health status $(P<$ 0.05). See Table 5.

\section{Discussion}

The concept of health proposed by the World Health Organization (WHO) is not only the absence of disease, but also comprised of the physical, psychological and social integrity ${ }^{[5-6]}$. Sub-health is a dynamic state that can lead to individual self-perceived decline of physical function, psychological function, and social adaptation function in varying degrees, without reaching any diagnostic criteria [7-8]. The sub-health state can cause many harms and seriously affect the quality of people's life and work. If no active intervention measures are taken, the sub-health state will cause long-term and hidden damage to the body, eventually leading to the occurrence of diseases ${ }^{[9]}$. Based on the WHO definition of health, $\mathrm{Xu}$ Jun and others proposed the operational definition of sub-health ${ }^{[4]}$, and conducted rigorous demonstrations through the Delphi expert consultation method ${ }^{[10]}$, item analysis and item screening ${ }^{[4]}$ and other methods, and 39 sub-health evaluations were finally selected. Then the first version of the SHMS V1.0 suitable for China's cultural background and social values was developed. This questionnaire has fewer entries, is easy to understand, and the survey is easy to carry out, with relatively high reliability and validity ${ }^{[11-15]}$, which provides an effective measurement tool for the determination of sub-health status.

Due to work intensity (physiological dimension), disease with high RO value and other unknown risks (psychological pressure), changes in personal and family life events of team members (social dimension), etc., we believe that while carrying out front-line treatment work, we also urgently need to pay attention to the health status of the medical team members aiding Wuhan. For this reason, we performed 
a preliminary study and found that the overall score of the sub-health assessment scale for the medical team aiding Wuhan Army was $(69.11 \pm 10.20)$ points, and the scores of the three subscales were: physiological subscale scores $(71.16 \pm 11.30)$ points, psychological subscale scores $(67.99 \pm 13.51)$ and the score of the social subscale $(67.41 \pm 12.31)$, which indicates that these medical staff are generally at a moderate sub-health level.

Many reports have shown that in the face of the invisible and intangible virus, the fear of the medical staff aiding Wuhan comes from the pneumonia infection or even death that may occur around them at any time. Coupled with irregular daily routine, the phenomenon of sub-health is generally not optimistic. Zhang Wei and others have investigated 155 first-line medical staff in Anhui Province during the COVID19 outbreak, and found that the incidence of psychological stress among medical staff assisting Wuhan was relatively high ${ }^{[16]}$. In another study, Chen Xia found that the mental health of medical staff from Xinjiang aiding Wuhan who worked in the quarantine area for 5-8 hours per day was more severely affected ${ }^{[17]}$.

Through single-factor and multi-factor analysis, it is found that women's mental sub-health level is significantly lower than that of men. This is similar to the results of most studies ${ }^{[18-19]}$. This may be due to women's physical characteristics and the burden of complexity from their multiple social roles, the resulting social and family pressures and more negative events have caused reduced women's health affected by many factors. Therefore, more health attention should be paid to women. Xiangdong Yuan et al. have concluded that the higher the education level, the less optimistic the sub-health status ${ }^{[20]}$. This study also found that the sub-health status of medical workers assisting Wuhan with different education levels also has significant differences, i.e. the higher the education level, the more serious the sub-health status is. This may be related to factors such as long-term sedentary study, infrequent participation in exercise, and greater academic pressure in high educated workers. Moreover, studies have shown that unhappiness in married life, divorce/widowed, etc. lead to decreased marriage satisfaction and mental health problems ${ }^{[21]}$. In this study, compared with unmarried and divorced, health status in those married, widowed, and unmarried cohabitation is more serious, which may be related to the smaller family responsibilities of unmarried and divorced people. It is suggested that maintaining good family relationships, understanding each other among members, and balancing life and working hours play important roles in maintaining family harmony.

The sub-health status of the medical staff who feel more work pressure is more serious than the staff who feel normal or less work pressure, indicating that the pressure has a certain impact on the sub-health status. Thus one should learn psychological counseling methods or talk to colleagues to relieve stress, at the same time, active participation in entertainment activities and group activities of hospitals or social organizations will help maintain a good mental state at all times. The sub-health level of only children is significantly higher than that of non-only children, and the sub-health level of central families is also significantly higher than that of extended families, indicating that family factors can impact sub-health. Some studies have found that negative event experiences can cause individuals to experience emotions 
such as restlessness, depression, and anxiety, which increase psychological pressure, endanger the healthy development physically and mentally, and even lead to anxiety, depression and tumors if they are affected by negative emotions for a long time ${ }^{[22-23]}$.

In this study, all of frustration, betrayal, and children's education problems can reduce the sub-health level. These negative events in life often make people feel depressed, and therefore they will be physically, psychologically, and socially adaptable.Good hobbies can edify people's sentiment and make people obtain multiple spiritual and physical happiness. From tour research, a wide range of hobbies can also have a positive impact on sub-health. Which is consistent with most studies ${ }^{[24-25]}$. Irregular diets and dietary bias can affect the sub-health status of medical workers assisting Wuhan. Therefore, scientific and reasonable work and rotation schedules should be formulated, and the work roles of medical staff should be appropriately adjusted. Consciously quit smoking and alcohol abuse addition to a reasonable diet are helpful to improve the health of medical staff.

\section{Conclusions}

In our study, we found that the medical staff aiding Wuhan are generally at a moderate sub-health level. Work pressure, only child, family structure, business frustration, hobbies, irregular diet, dietary bias, betrayal, and children's education problems are the main influencing factors of sub-health. Therefore, we should work together from multiple perspectives of individuals, families, and society, take effective intervention measures to strengthen health management, and actively promote the transformation of sub-health status to healthy status, so as to effectively improve the ability of continuous medical rescue and provide support for complete epidemic prevention and control tasks with high quality.

\section{Abbreviations}

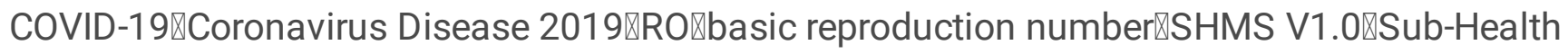
Measurement Scale Version 1.0 $₫$ WHO:World Health Organization

\section{Declarations}

\section{Funding}

This research was in part supported by the Special Scientific Research Project of Military Commission Health Care (18BJZ11), the Special Project of Innovation and Generation of Military Medical Support Capability (20WQ020), National Natural Science Fund, Foundation of China (No: 71673126), Science and Technology Planning Project of Guangzhou City of China (No: 201803010089), Philosophy and Social Sciences of Guangdong College for the project of "Public Health Policy Research and Evaluation" Key Laboratory(No.2015WSY0010), and Public Health Service System Construction Research Foundation of Guangzhou(2018-2020).

\section{Availability of data and materials}


The datasets used and/or analyzed during the current study are available from the corresponding author on reasonable request.

\section{Author's contributions}

Meixian Zhang and Haoyuan Chen are the primary investigator of this study and they also executed the experiments. Xia Li designed the main idea and undertook data interpretation, manuscript writing, and revision, Dihui Xu and Jie Li participated in the design of this research. Chen Huang and Qian Liu conducted statistical analyses, Jun Xu developed Sub-Health Measurement Scale Version 1.0 (SHMS V1.0) .

\section{Competing interests}

The authors declare that they have no competing interests.

\section{Ethical Approval and Consent to participate}

Not applicable

\section{Authors' information}

Not applicable

\section{Consent for publication}

Not applicable

\section{References}

1. World Health Organization. Surveillance case definitions for human infection with novel coronavirus (nCoV). 2020.

2. Alimohamadi Y, Taghdir M, Sepandi M. Estimate of the Basic Reproduction Number for COVID-19: A Systematic Review and Meta-analysis. J Prev Med Public Health. 2020;53:151-157.

3. Zhang JH, Xu J, Huang JM, Feng LY, Luo R. Quantitative Measurement of Sob-health Research. Guangdong Med J. 2009;30:1746-1748.

4. Xu J, Lu Y, Feng LY, Qiu JC, Zou LA, Qu RJ, et al. Preliminary study of Sub-Health Measurement Scale Version1.0 Norms for Chinese Civil Servants. J South Med Univ. 2011;31:1654-1662.

5. Su JJ, Zhang DQ. An Historical Study on the Development of WHO Health Definitions. Chin J Hist Sci Technol. 2016;37:485-496.

6. Li XL, Zhao GC, Xu YL. Advance in Research of Sub-health (review). Chin J Rehabilitation Theor Pr. 2008;14:942-946. 
7. China Association of Chinese Medicine. Clinical guidelines of Chinese Medicine on Sub-health, vol. 10. Beijing: China Press of Traditional Chinese Medicine; 2006.

8. Zhu R. Interpretation of Clinical guidelines of Chinese Medicine on Sub-health. Chinese Medicine Modern Distance Education of China. 2009;8:79-80.

9. Zheng RH. Main Influencing Factors and Prevention Measures of Sub-health. Health Vocational Educ. 2008;26:145-146.

10. Chen QS, Wang SY, Jing CX, Dong XM, Chi GB, Zhu L. Application of Delphi Method to Evaluate the Diagnostic Criteria of Sub-health. Chin J Public Health. 2003;19:1467-1468.

11. Lu Y, Xu HL, Wei Q, Xu J. The Confirmatory Factor Analysis of Sub-health Rating Scale Applied to Urban Residents. Chin Gen Pr. 2013;13:1108-1109.

12. Lin YQ, Xu J, Li WY, Wu WX, Xia C. Reliability and Validity of Sub-health Measurement Scale Version 1.0 for Evaluating the Sub-health Status of Anhui Urban Residents. Chin Gen Pr. 2018;21:1845-1851.

13. Cai YJ, Lu Y, Xu J, Xie J. Reliability and Validity Research of Sub-health Measurement Scale Version 1.0 Applied in Chaozhou Town Community Residents. J Pr Med. 2013;29:126-128.

14. Qu RJ, Yang YB, Feng LY, Xu J. Reliability and Validity of SHMS V 1.0 in Evaluating Sub-health Condition of Xinjiang Civil Servants. Chin Gen Pr. 2012;15:744-747.

15. Zhong CD, Xie J, Xu J, Feng LY. Health Status of Civil Servants in Tianjin and Reliability and Validity of Sub-Health Measurement Scale Version 1.0 (SHMS V1.0). Chin J Public Health. 2012;28:13481351.

16. Zhang W, Jiang HJ, Jiang XG, Zhang XC, Wu YD, Li KK, et al. Analysis of Psychological Stress and Influencing Factors of of Anhui's Medical Staff Assisting Hubei Under Novel Coronavirus Pneumonia. J Changzhi Med Coll. 2020;34:92-96.

17. Chen X, Wu X, Hou XX, Wang HC. Mental Health Status among Medical Staff from Xinjiang during COVID-19 Epidemic Control in Hubei Province: a Cross-sectional Survey. Chin J Public Health. 2020;36:682-685.

18. Wang WM, Huang ZY, Zhang P, Xu T, Tong WP, Shan GL. Prevalence of Sub-health and its Influencing Factors in Residents of Suzhou City. Chin J Public Health. 2013;29:325-327.

19. Yuan $P$, Tang YL, Yu XF. Sub-health and its Influencing Factors among Residents of Jiangmen City. Chin J Public Health. 2012;28:844-847.

20. Yuan XD, Zhang X, Lv JJ, Zhang JX, Gu P, Zou GL, et al. Research on Sub-health Status and Influencing Factors of Some Occupational Population in Guangzhou. J South Med Univ. 2007;27:1548-1550.

21. Tian MM, Chen CX, Li SX, Ma SH. Influentces of Family and Social Support on Mental Health among Elderly Residents in Hebei Province. Chin J Public Health. 2015;31:156-159.

22. Zhong CD. Study on Sub-health State and Associated Factors among Civil Servants in Tianjin. Tianjin: Tianjin Medical University; 2012. 
23. Xie YM, Liu BY, Pu HY, He LY, Yi DH, Li WQ, et al. Analysis of Risk Factors of Sub-health Status in Different Populations in Beijing Area. Chin J Public Health. 2006;22:1106-1107.

24. Lu Y. Sub-health and Associated Factors of Guangdong Town Dweller. Guangzhou: Southern Medical University; 2013.

25. Li Q, Zhang XM, Li JH, Hu M. Investigation and Analysis of Sub-health Influencing Factors in Community Inhabitants of Shenyang City. Med Soc. 2010;23:33-35.

\section{Tables}

Due to technical limitations, table 1, 2, 3, 4 and 5 is only available as a download in the Supplemental Files section.

\section{Supplementary Files}

This is a list of supplementary files associated with this preprint. Click to download.

- Table1to5.docx 